\title{
Mesialização de molares com ancoragem em mini-implantes
}

\author{
Marcos Janson*, Daniela Alcântara Fernandes Silva**
}

\begin{abstract}
Resumo
Introdução: é muito comum, na rotina do consultório odontológico, especialmente em Ortodontia, o paciente, após avaliar o plano de tratamento proposto, questionar sobre a possibilidade de fechamento de espaços presentes, ocasionados pela perda de dentes permanentes. O ortodontista, frente a essa questão, tem que avaliar diversos fatores, tais como a má oclusão presente, a integridade do osso e das raízes, o tempo de tratamento e a geometria do posicionamento dentário, que é o que permite avaliar se, com o fechamento dos espaços e, conseqüentemente, a perda de ancoragem dos segmentos anteriores, a finalização estará de acordo com os ideais que regem a especialidade. Com o advento dos mini-implantes, as possibilidades desta abordagem terapêutica aumentaram, pois o efeito adverso da perda de ancoragem pode ser eliminado e, com isso, o paciente pode ser beneficiado. No entanto, os outros fatores envolvidos, assim como a mecânica do movimento, requerem especial atenção. Objetivos: no presente artigo serão abordados os fatores envolvidos neste tipo de tratamento, o raciocínio na tomada de decisões e os detalhes importantes que devem ser observados durante a condução da mecânica, ilustrados com casos clínicos.
\end{abstract}

Palavras-chave: Ancoragem absoluta. Ancoragem esquelética. Mini-implantes. Mesialização de molares.

\section{INTRODUÇÃO}

A mesialização de molares com ancoragem esquelética consiste no movimento mesial destes dentes sem que haja reações nos segmentos mais anteriores do arco. O fechamento dos espaços de primeiros molares perdidos precocemente deve ser realizado com movimento de corpo dos segundos molares e, às vezes, também dos terceiros, o que significa deslocamento anterior que varia de 12 a $15 \mathrm{~mm}$.

\section{INDICAÇÕES}

Freqüentemente, este tipo de movimento visa o fechamento de espaços anteriores aos molares, ocorridos devido a fatores diversos e que, para não comprometer o perfil ou devido ao posicionamento geométrico dos demais dentes, não podem ser fechados da maneira convencional, ou seja, com a retração dos dentes anteriores. Dentre as indicações destacam-se:

- Fechamento de espaços edêntulos decorren-

\footnotetext{
* Especialista e Mestre em Ortodontia pela FOB-USP Bauru. Professor e Coordenador do Curso de Especialização em Ortodontia da ABCD-Bahia.
}

** Especialista em Ortodontia pela ABCD-Bahia. Professora do Curso de Especialização em Ortodontia da ABCD-Bahia. 
tes de perda dos dentes permanentes (caso 1): esta é a indicação mais comum. O paciente, geralmente adulto, apresenta-se para tratamento e, ciente das informações a respeito da possibilidade de movimentação dentária no sentido ântero-posterior, questiona a pertinência do fechamento, para que não seja necessária a reabilitação com próteses ou implantes.

- Fechamento de espaços devido a agenesias (caso 2): as agenesias de dentes posteriores, especialmente os pré-molares, podem ser solucionadas de diversas maneiras, tais como: na presença de Classe I com biprotrusão ou apinhamento dentário, podem ser extraídos os outros dentes equivalentes em cada hemiarco e fechados os espaços ou, então, o mesmo protocolo pode ser utilizado quando há protrusão dentoalveolar e a retração dos dentes anteriores beneficiaria a estética facial. No entanto, quando não há protrusão dentária ou a agenesia está presente no maxilar deficiente, como, por exemplo, na mandíbula nos casos de Classe II ou maxila nos casos de Classe III, a redução da massa dentária não pode ocorrer e, portanto, o espaço da agenesia deve ser preservado, sendo indicada a restauração com próteses convencionais ou implantes.

- Extração de dente permanente de prognóstico duvidoso (caso 3): no planejamento de um caso clínico, muitas vezes, o ortodontista se depara com dentes que apresentam problemas endodônticos, cáries extensas, infiltrações em próteses e perdas ósseas verticais ou horizontais. Nestes casos, o paciente pode ser beneficiado com a extração deste dente e subseqüente mesialização dos dentes mais posteriores, preservando, desta maneira, somente os dentes saudáveis e reduzindo os custos de outras intervenções odontológicas.

\section{FATORES ENVOLVIDOS NO DIAGNÓSTICO Tempo de tratamento}

Deve ser o primeiro parâmetro a ser avaliado, pois a mesialização de molares prolonga o tempo de tratamento, em média, 1 ano, desde que todas as condições sejam favoráveis. De acordo com Roberts et al. ${ }^{8,10}$, no movimento dos segundos e terceiros molares, com ancoragem esquelética, a taxa de movimentação é de, aproximadamente, $0,5 \mathrm{~mm} / \mathrm{mês}$, que corresponde à taxa linear da reabsorção osteoclástica ${ }^{9}$. Dessa forma, é conveniente prever um prazo de tratamento de, aproximadamente, 3 anos para a finalização dos casos.

Ou seja, a indicação de aparelho ortodôntico, somente para realizar este tipo de tratamento, não é bem indicada, pois a reabilitação com implantes tomaria um tempo menor e com um custo equivalente. Portanto, o movimento é bem indicado quando há outras necessidades ortodônticas.

\section{Movimentação em rebordo atrófico}

A presença do osso alveolar é justificada enquanto o dente está presente, para servir de sustentação. Uma vez que o dente é perdido, o osso alveolar, não apresentando mais função, se atrofia, tanto no sentido vestíbulo-lingual quanto em altura. Portanto, o ortodontista, ao planejar o fechamento de espaços edêntulos, deve estar ciente de como o osso atrófico se comporta em relação à movimentação ortodôntica. Para que se possa visualizar os resultados que podem ser alcançados, dois efeitos importantes da fisiologia da movimentação dentária devem ser entendidos. O primeiro é a possibilidade de movimentação do dente em direção a áreas atróficas. Alguns autores $^{3,10,11}$ já demonstraram, em jovens e adultos, a possibilidade de fechamento de espaços de primeiros molares perdidos há muito tempo, que apresentavam rebordo atrófico. Concluiu-se que, durante o fechamento de espaços de até $10 \mathrm{~mm}$, o rebordo ósseo acompanha o dente, mais precisamente o movimento mesial do segundo molar. Salienta-se, entretanto, que, em indivíduos adultos, parece haver uma maior tendência de reabsorção da crista alveolar, aproximadamente $2 \mathrm{~mm}$, sem que, com isso, ocorra prejuízo ao periodonto. Nestes trabalhos, não foram analisados os parâmetros periodontais de perda de inserção. 
De acordo com Fontenelle ${ }^{1}$, os dentes podem ser movimentados com o osso ou através do osso e a pré-condição para que isto ocorra é haver reabsorção direta na direção do movimento. Quando os dentes se movimentam através do osso, o mecanismo é de reabsorção indireta, a qual não é acompanhada de aposição óssea. Este processo ocorre quando as forças são demasiadamente intensas, a ponto de causar hialinização do ligamento periodontal. Segundo Roberts et al. ${ }^{10}$, não pode haver pressão no periósteo que impeça o fluxo sangüíneo para os osteoblastos. Desta forma, a movimentação do dente para áreas de perda óssea ou onde a forma do rebordo alveolar é mais estreita é possível, desde que um sistema de forças adequado seja planejado ${ }^{7}$.

A segunda é a resposta tecidual que ocorre quando se movimenta o dente para áreas onde a altura do rebordo ósseo é menor. A idéia que se tinha da movimentação dentária sempre foi da criação de duas zonas distintas: uma de tensão, que provocaria deposição óssea, e outra de pressão, que acarretaria reabsorção do osso frontal ao movimento. No entanto, Thilander ${ }^{12}$ e Geraci et al. ${ }^{2}$ demonstraram que, quando o movimento é realizado lentamente e a higiene bucal é adequa$\mathrm{da}$, apesar de haver o estreitamento da crista do lado de pressão, não há perda de inserção e tanto o nível ósseo como o gengival mantêm-se intactos. Assim, na face frontal ao rebordo, o periodonto acompanhará o movimento do dente, ocorrendo um estreitamento da crista, porém sem perda de inserção, e do lado de tensão haverá neoformação óssea da mesma altura e largura do dente que está sendo movimentado. Ao final, o dente ocupará o espaço onde o osso era atrofiado, sem prejuízo ao seu periodonto.

Ciente dessas considerações, a pergunta que comumente se faz é sobre qual grau de atrofia vestíbulo-lingual contra-indicaria o movimento. Não há trabalhos quantificando esta informação, no entanto, Kokich ${ }^{6}$, empiricamente, acredita que quando o osso na mesial do molar apresenta es- pessura de, pelo menos, metade da coroa, o movimento pode ser realizado com pouco risco.

\section{Qualidade e quantidade da gengiva nos dentes que serão movimentados}

A recessão secundária na superfície vestibular da raiz mesiovestibular do segundo molar inferior pode, algumas vezes, ocorrer. Os fatores que, provavelmente, estão relacionados são: a) a presença de uma deiscência subjacente no osso sobre a raiz mesiovestibular do molar. Se uma deiscência estiver presente, é provável que ocorra uma recessão durante o fechamento de espaço; b) o tipo e quantidade de tecido gengival que está presente em cada paciente. Se um paciente apresentou um biotipo de tecido espesso, então isto poderia oferecer proteção ao dente, prevenindo a recessão. Porém, se o paciente tem uma zona estreita de gengiva e tem um biotipo de tecido fino, a recessão pode ser mais provável durante o fechamento do espaço ${ }^{6}$. O mais seguro é constatar a presença de, pelo menos, $2 \mathrm{~mm}$ de gengiva ceratinizada na vestibular da raiz mesial.

\section{CONSIDERAÇÕES MECÂNICAS}

A mesialização dos molares é considerada um movimento de corpo e é melhor conduzida quando as raízes encontram-se verticalizadas. Deste modo, antes de se iniciar a mesialização, a verticalização dos molares ${ }^{4,5}$ é necessária. Após esta fase, iniciase o movimento, que pode ser conduzido em fio redondo de calibre 0,020 " ou retangular de 0,018 " x 0,030 " em slot 0,022 ". O fato de se trabalhar com fio redondo ou retangular de calibre reduzido explica-se devido ao movimento não necessitar de controle de torque vestíbulo-lingual e para facilitar o deslize do braquete no fio. Por ser muito extenso, há uma tendência de inclinação dos molares durante o movimento, portanto, adota-se um protocolo de ativação que consiste na ativação mesial, durante 3 ou 4 meses, com intervalos de 2 meses, nos quais o dente é, novamente, verticalizado, por meio da recolagem dos tubos ou dobras nos fios. 
Uma alternativa que facilita a mecânica, diminuindo a inclinação durante a translação do dente, é a incorporação de um braço de força por vestibular, lingual ou ambas.

\section{POSIÇÃO DOS MINI-IMPLANTES}

Os mini-implantes, preferencialmente, devem ser instalados o mais próximo possível do plano oclusal, pois assim diminui-se o vetor intrusivo na mesial do molar e, conseqüentemente, sua inclinação. A colocação de mini-implantes por vestibular e palatino também é mais adequada, pois elimina a rotação dos dentes observada quando se utiliza somente um ponto de apoio (Fig. 2D).
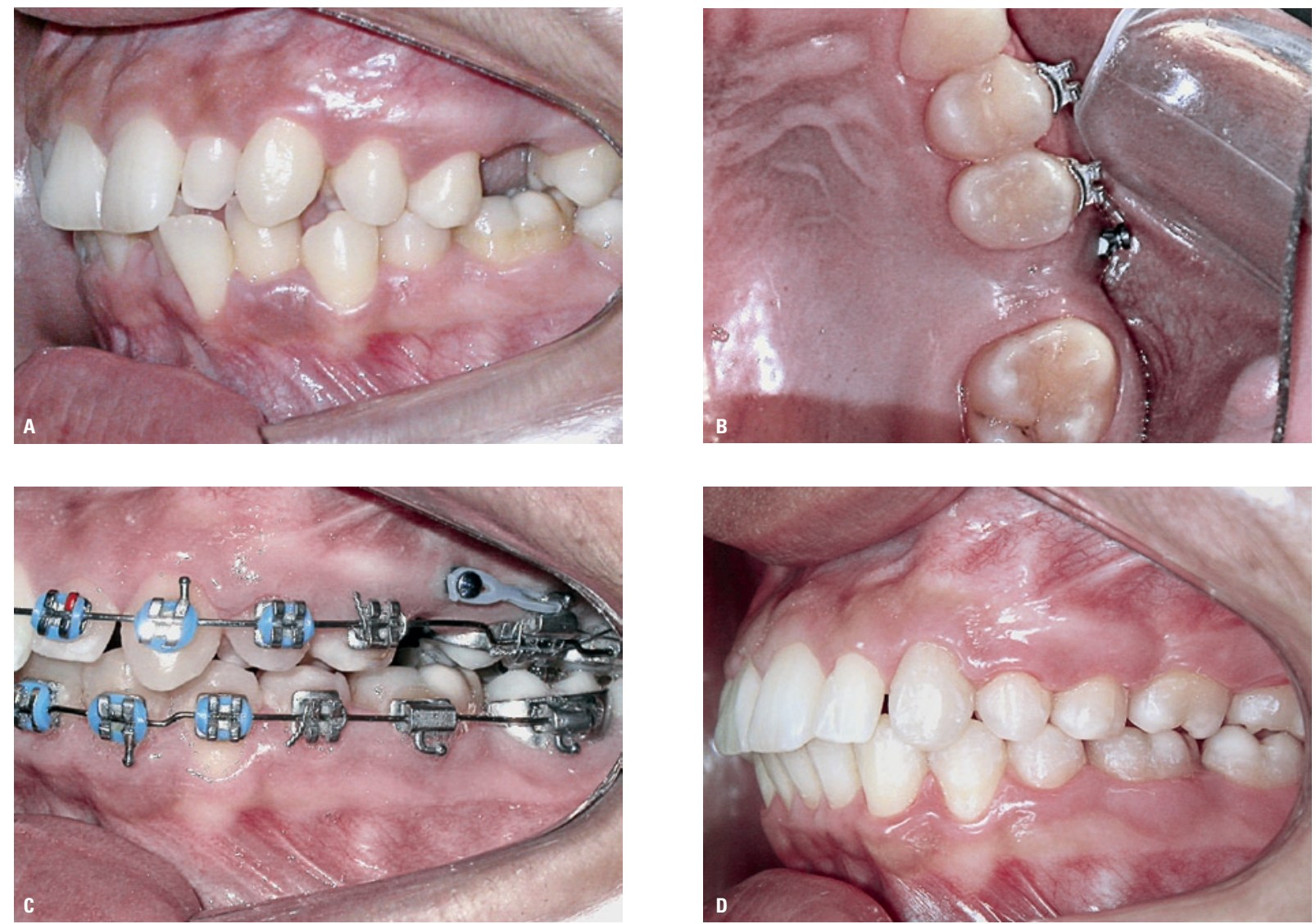

FIGURA 1 - A) Foto lateral esquerda, onde se nota a má oclusão de Classe I e o espaço edêntulo do primeiro molar superior. B) Foto oclusal antes do início do fechamento do espaço, com o mini-implante localizado pela vestibular. C) Foto lateral esquerda durante a mesialização dos molares. D) Foto final, com os espaços fechados e a manutenção do relacionamento dentário de Classe I. 

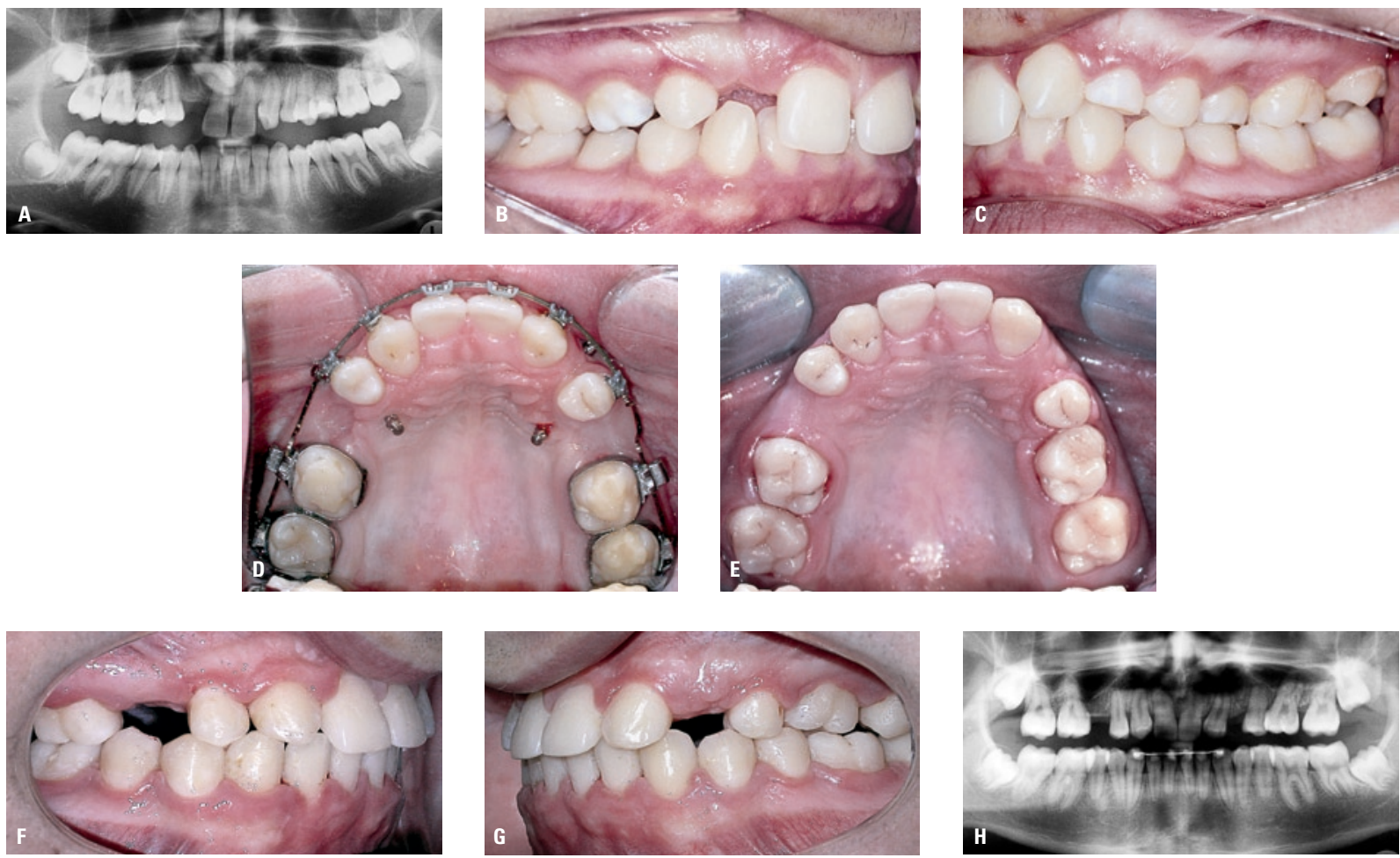

FIGURA 2 - A) Radiografia panorâmica onde se nota o canino superior direito não irrompido e agenesia dos incisivos laterais e segundos pré-molares superiores

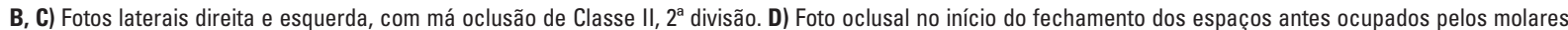
decíduos. Note os mini-implantes posicionados por vestibular e palatino. E) Foto oclusal ao final do tratamento. 0 espaço do segundo pré-molar superior direito não pôde ser fechado, devido à anquilose manifestada pelo primeiro molar, que impossibilitou seu movimento para mesial. F, G) Fotos laterais direita e esquerda ao final do tratamento. Os espaços foram preparados para a instalação de implantes. H) Radiografia panorâmica final. Note que há reabsorções apicais generalizadas, fator este que já era previsto, devido ao formato das raízes na radiografia inicial.

$2^{a}$ divisão, canino superior direito não irrompido e agenesia dos incisivos laterais e segundos prémolares superiores. Foi planejado o tracionamento do dente 13, extração dos molares decíduos e subseqüente fechamento dos espaços. Quanto às agenesias dos laterais, os caninos os substituiriam e, em suas posições, seria preparado o espaço para a colocação de implante osteointegrado do lado esquerdo e, do lado direito, o primeiro pré-molar substituiria o canino e o espaço distal a este seria fechado. Devido à anquilose manifestada pelo primeiro molar superior direito, permaneceu o espaço do segundo pré-molar, que também seria reconstituído com implante.

CASO 3 - Paciente com 24 anos de idade, gênero feminino, má oclusão de Classe II, $1^{\text {a }}$ divisão.
O planejamento proposto foi a extração dos dois primeiros pré-molares superiores, no entanto a paciente relatou o desejo de extrair o primeiro molar inferior esquerdo, que apresentava prognóstico duvidoso, devido a problemas na coroa e defeito ósseo na parede vestibular. Dessa forma, foi proposta a mesialização dos segundo e terceiro molares inferiores com ancoragem esquelética (Fig. 3).

\section{CONCLUSÕES}

Como pôde-se observar, por meio dos casos descritos, a mesialização de molares é um recurso extremamente útil na clínica ortodôntica e beneficia primariamente o paciente, pois diminui a necessidade de substituição de dentes perdidos ou 

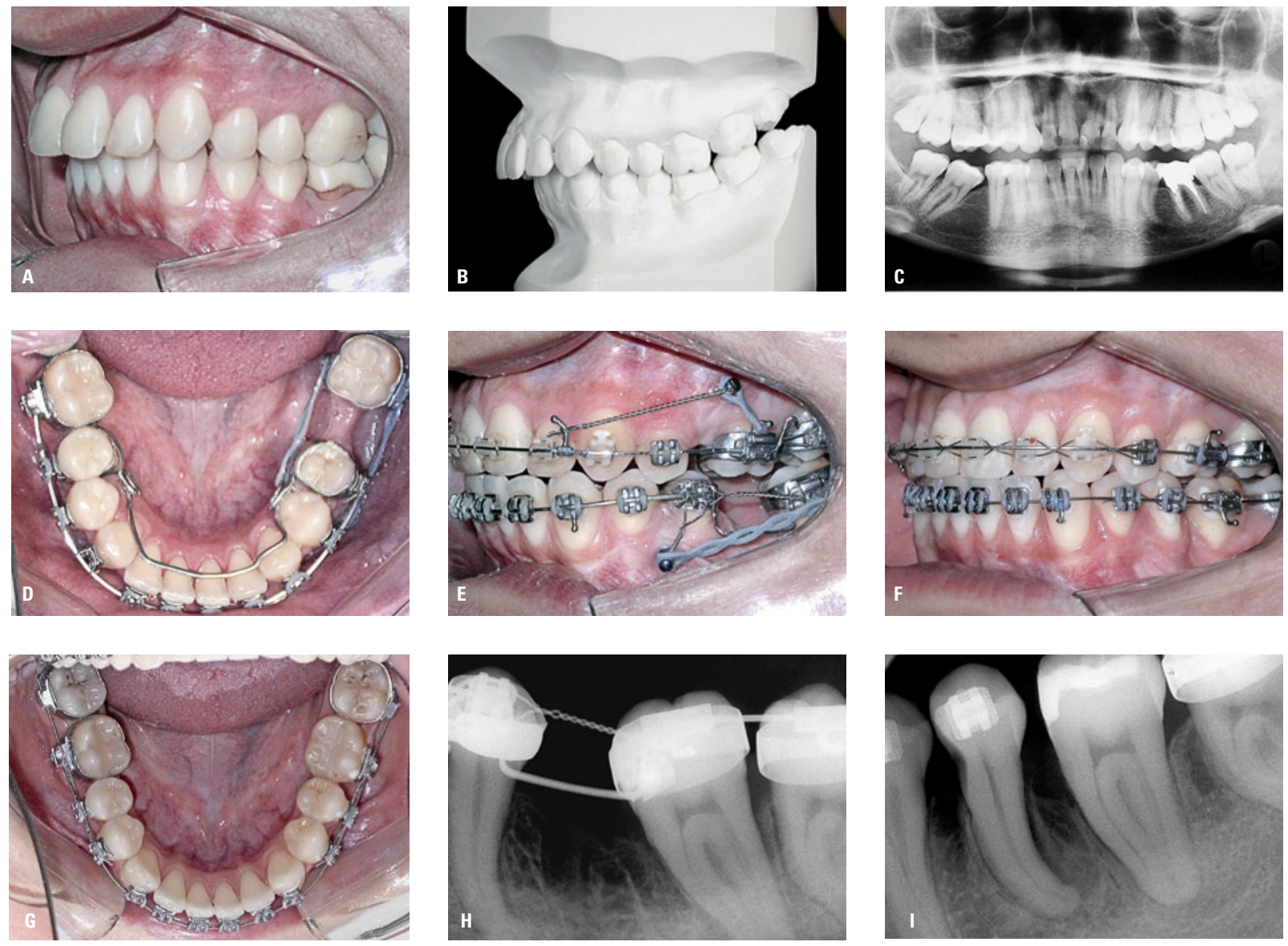

FIGURA 3 - A, B) Fotos intrabucal e do modelo do lado esquerdo, onde observa-se a má oclusão de Classe Il completa e curva de Spee bastante acentuada. C) Radiografia panorâmica inicial. D) Foto oclusal no início da mesialização dos molares. Note o rebordo extremamente atrófico após a extração do primeiro molar inferior esquerdo. E) Foto lateral esquerda durante os movimentos ântero-posteriores com mini-implantes superiores. F) Foto lateral esquerda após o fechamento dos espaços. Note 0 relacionamento de Classe I alcançado nos caninos e a recessão gengival na raiz do molar. Um enxerto gengival livre foi programado para esta área. G) Foto oclusal após o fechamento dos espaços. H, I) Radiografias periapicais ao início e ao final do tratamento.

ausentes por próteses. No entanto, o movimento demanda maior tempo de tratamento, a mecânica induz alguns efeitos colaterais que devem ser contrapostos durante o movimento (como a inclinação e extrusão dos molares) e fatores envolvidos na qualidade do rebordo atrófico devem ser ponderados, para se evitar efeitos indesejados. Quando for planejado este tipo de movimento, todas as opções disponíveis devem ser expostas ao paciente, para que ele possa, junto com o pro- fissional, fazer sua melhor escolha, ponderando os custos financeiros, tempo de tratamento e previsibilidade dos resultados. 


\title{
Mesial movement of molars with mini-implants anchorage
}

\begin{abstract}
Introduction: It's routine, especially at the orthodontic office, the patient, after the assessment of the proposed treatment plan, answer about the possibility of closing their edentulous spaces caused by missed permanent teeth. In these situations, several factors must be evaluated, like the present malocclusion, the bone and roots integrity, the treatment time and the geometry of teeth positioning, that is what permits to assess if the loss of anchorage from the anterior segments, during the space closure, would permit the occlusion to end within the orthodontic ideals. With the mini-implants, the possibilities of this therapeutic approach have been improved, since the adverse effects are eliminated with the skeletal anchorage. Aim: In the present paper will be analyzed the factors involved in this treatment type, the reasoning in the decisions making and the important details that should be observed during the mechanics conduction, illustrated by cases reports.
\end{abstract}

Key words: Absolute anchorage. Skeletal anchorage. Mini-implants. Molar mesial movement.

\section{REFERÊNCIAS}

1. FONTENELLE, A. Limitations in adult Orthodontics. In: MELSEN, B. (Ed.). Current controversies in Orthodontics. Chicago: Quintessence, 1991. p. 147-179.

2. GERACI, T. F.; NEVINS, M.; CROSSETTI, H. W.; DRIZEN, K.; RUBEN, M. P. Reattachment of the periodontium after tooth movement into an osseous defect in a monkey. Int. J. Periodontics Restorative Dent., Chicago, v. 10, no. 3, p. 184 197, 1990.

3. HOM, B.; TURLEY, P. The effects of space closure of the mandibular first molar areas in adults. Am. J. Orthod.

Dentofacial. Orthop., St. Louis, v. 85, no. 6, p. 457-469, 1984.

4. JANSON, M. Verticalização de molares. In: _. Ortodontia em adultos e tratamento interdisciplinar. Maringá: Dental Press, 2008. p. 129-167.

5. JANSON, M. R. P.; JANSON, R. R. P.; MARTINS, P. F. Tratamento interdisciplinar I: considerações clínicas e biológicas na verticalização de molares. Rev. Dental Press Ortodon. Ortop. Facial, Maringá, v. 6, no. 3, p. 87-104, 2001

6. KOKICH, V. Entrevista Dr. Vincent Kokich. Rev. Dental Press Ortodon. Ortop. Facial, Maringá, v. 11, n. 6, p. 19-23, 2006.

7. MELSEN, B. Current controversies in Orthodontics. Chicago: Quintessence, 1991.
8. ROBERTS, W. E.; ARBUCKLE, G. R.; ANALOUI, M. Rate of mesial translation of mandibular molars using implant-anchored mechanics. Angle Orthod., Appleton, v. 66, no. 5, p. 331-338, 1996.

9. ROBERTS, W. E.; GOODWIN, W. C. J.; HEINER, S. R. Cellular response to orthodontic force. Dent. Clin. North Am. Philadelphia, v. 25, no. 1, p. 3-17, 1981.

10. ROBERTS, W. E.; MARSHALL, K. J.; MOZSARY, P. G. Rigid endosseous implant utilized as anchorage to protract molars and close an atrophic extraction site. Angle Orthod. Appleton, v. 60, no. 2, p. 135-152, 1990

11. STEPOVICH, M. A clinical study of closing edentulous spaces in the mandible. Angle Orthod., Appleton, v. 49, no. 4, p. 227233,1979

12. THILANDER, B. Infrabony pockets and reduced alveolar bone height in relation to orthodontic therapy. Semin Orthod. Philadelphia, v. 2, no. 1, p. 55-61, 1996.
Endereço para correspondência

Marcos Janson

Rua Saint Martin 22-23, Bauru/SP

CEP: 17.043-080

E-mail: jansonm@uol.com.br 